\title{
EXACT VALUE OF THE GROUND STATE ENERGY OF THE LINEAR ANTIFERROMAGNETIC HEISENBERG CHAIN WITH NEAREST AND NEXT-NEAREST NEIGHBOUR INTERACTIONS
}

\author{
P.M. van den BROEK \\ Department of Applied Physics, Twente University of Technology, Enschede, The Netherlands
}

Received 4 March 1980

It is shown that the ground state energy of the hamiltonian $H=\Sigma S_{i} \cdot S_{i+1}+\gamma \Sigma S_{i} \cdot S_{i+2}$ for the linear antiferromagnetic Heisenberg chain with nearest and next-nearest neighbour interactions is equal to $-\frac{3}{2}$ if $\gamma=\frac{1}{2}$.

We consider the linear antiferromagnetic Heisenberg chain with nearest and next-nearest neighbour interactions with hamiltonian

$H_{N}(\gamma)=\sum_{i=1}^{N-1} S_{i} \cdot S_{i+1}+\gamma \sum_{i=1}^{N-2} S_{i} \cdot S_{i+2}$,

where $N$ is the number of spins $\frac{1}{2}$ in the chain. Let the lowest eigenvalue of $H_{N}(\gamma)$ be $E_{N}(\gamma)$. We are interested in the energy per spin in the ground state in the limit of large $N$ :

$E(\gamma)=\lim _{N \rightarrow \infty} E_{N}(\gamma) / N$

$E(0)$ is known exactly $[1,2]$; it is equal to $1-4 \ln 2$ $(=-1.7726, \ldots)$. For $\gamma \neq 0$ only approximations and upper and lower bounds for $E(\gamma)$ are knwon [3-8]. Majumdar and Ghosh $[3,4]$ have found that for small finite chains $(N \leqslant 10)$ with an even number of spins and periodic boundary conditions $E_{N}\left(\frac{1}{2}\right)$ is equal to $-\frac{3}{2} N$. Moreover they showed that $-\frac{3}{2} N$ is an eigenvalue of $H_{N}\left(\frac{1}{2}\right)$ for all even $N$. The same is true for open chains with $N$ even [5]. Thus $E\left(\frac{1}{2}\right) \leqslant-\frac{3}{2}$. The aim of this note is to show that $E\left(\frac{1}{2}\right)$ is equal to $-\frac{3}{2}$ and that $E(\gamma)$ takes its maximal value at $\gamma=\frac{1}{2}$.

Divide the chain into $\frac{1}{2} N$ cells of 2 spins. Let the state $|\psi\rangle$ of the chain be the direct product of the states. $2^{-1 / 2}(|+-\rangle-|-+\rangle)$ for the cells. Then it is easy to verify that

$$
H_{N}\left(\frac{1}{2}\right)|\psi\rangle=-\frac{3}{2} N|\psi\rangle,
$$

and that

$\left\langle\psi\left|H_{N}(\gamma)\right| \psi\right\rangle=-\frac{3}{2} N$.

Therefore

$E(\gamma) \leqslant-\frac{3}{2}$.

This shows that $E(\gamma)$ takes its maximal value $-\frac{3}{2}$ at $\gamma=\frac{1}{2}$ if we show that $E\left(\frac{1}{2}\right)=-\frac{3}{2}$. To show that $E\left(\frac{1}{2}\right)=$ $-\frac{3}{2}$ we calculate a lower bound for $E(\gamma)$.

The hamiltonian $H_{N}$ can be written as

$H_{N}(\gamma)=\sum_{i=1}^{N-2} H_{i}(\gamma)+\frac{1}{2} S_{1} \cdot S_{2}+\frac{1}{2} S_{N-1} \cdot s_{N}$,

where

$H_{i}(\gamma)=\frac{1}{2} S_{i} \cdot S_{i+1}+\frac{1}{2} S_{i+1} \cdot S_{i+2}+\gamma S_{i} \cdot S_{i+2} \cdot$

Since, in general, the lowest eigenvalue of a sum of operators is not less than the sum of the lowest eigenvalues of these operators, the lowest eigenvalue of $H_{N}(\gamma)$ is not less than $(N-2) E_{i}(\gamma)-3$ if $E_{i}(\gamma)$ is the lowest eigenvalue of $H_{i}(\gamma) . E_{i}(\gamma)$ can be calculated immediately; we find

$$
\begin{array}{rlrl}
E_{i}(\gamma) & =\gamma-2, & & \text { if } \gamma \leqslant \frac{1}{2}, \\
& =-3 \gamma, \quad & \text { if } \gamma \geqslant \frac{1}{2} .
\end{array}
$$

Since $E_{i}\left(\frac{1}{2}\right)=-\frac{3}{2}$ it follows that $E_{N}\left(\frac{1}{2}\right) \geqslant-\frac{3}{2} N$. It 
follows that $E\left(\frac{1}{2}\right) \geqslant-\frac{3}{2}$ which proves that $E\left(\frac{1}{2}\right)=$ $-\frac{\mathbf{3}}{\mathbf{2}}$.

It is a pleasure to acknowledge some useful discussions with Prof. Dr. W.J. Caspers.

\section{References}

[1] H. Bethe, Z. Phys. 71 (1931) 205.

[2] L. Hulthén, Ark. Mat. Astron. Fys. 26A (1938) No. 11.
[3] C.K. Majumdar and D.P. Ghosh, J. Math. Phys. 10 (1969) 1388

[4] C.K. Majumdar and D.P. Ghosh, J. Math. Phys. 10 (1969) 1399.

[5] P.M. van den Broek, W.J. Caspers and M.W.M. Willemse, submitted to J. Stat. Phys.

[6] Th. Niemeyer, J. Math. Phys. 12 (1971) 1487.

[7] H.P. van de Braak, W.J. Caspers, C. de Lange and M.W.M. Willemse, Physica 87A (1977) 354.

[8] H.P. van de Braak, W.J. Caspers, P.K.H. Gragert and M.W.M. Willemse, J. Stat. Phys. 20 (1978) 577. 